\title{
Quantifying the Sensitivity of Experimental Data to Variations in the QGP Equation of State
}

\author{
R.A. Soltz ${ }^{* \dagger}$ \\ Lawrence Livermore National Laboratory \\ E-mail: soltz@llnl.gov
}

We have developed a framework, the Comprehensive Heavy Ion Model Evaluation Reporting Algorithm (CHIMERA) to determine the properties of the Quark Gluon Plasma using chi-squared evaluations of models and data. We have used this framework to study simple participant and binary collisions scaling in the presence of pre-equilibrium flow in the context of the VH2 2D+1 viscous hydrodynamic model with UrQMD afterburner for data from RHIC. Here we present results from using this framework to explore variations in the equation of state.

8th International Workshop on Critical Point and Onset of Deconfinement,

March 11 to 15, 2013

Napa, California, USA

* Speaker.

${ }^{\dagger}$ A footnote may follow. 


\section{Introduction}

Since the initial success in applying hydrodynamic models to heavy ion collisions at the Relativistic Heavy Ion Collider (RHIC) and more recently from the Large Hadron Collider (LHC), we have made enormous progress in understanding the various stages of a heavy ion collision, from pre-equilibrium stage, through the hydrodynamic evolution and freeze-out via hadronic transport. In particular, the treatment of the hydrodynamic stage is now quite advanced, incorporating shear viscosity $[1,2]$ and initial energy density fluctuations in the initial conditions [3-5]. The success of these models in reproducing the general features of particle spectra and elliptic flow has led to the conclusion that the QCD matter created in relativistic heavy ion collisions behaves very much like a fluid with small shear viscosity to entropy ratio. However, the comparisons to spectra and anisotropic flow tend to follow a set prescription, and seldom include rigorous statistical evaluations.

In this paper we describe such an evaluation, with an emphasis on the sensitivity to the equation of state, with the ultimate goal to fully constrain the properties of the quark gluon plasma. We have developed a Comprehensive Heavy Ion Model Evaluation and Reporting Algorithm (CHIMERA) for systematically comparing a set of hybrid hydrodynamic models for heavy ion collisions spanning a range of initial parameters [6]. This framework enables one to determine the optimal parameters and associated uncertainties that best describe a set of soft physics measurements, incorporating both statistical and systematic errors. In the current implementation we use the $2 \mathrm{D}+1$ viscous hydro code VH2 [1] augmented with initial state eccentricity fluctuations [7] and pre-equilibrium flow [8] to describe the hydrodynamic evolution, and the Ultra-relativistic Quantum Molecular Dynamics hadronic cascade code (UrQMD) $[9,10]$ to describe the hadronic transport. To compare to data we generate particle spectra and elliptic flow directly from the UrQMD output. We generate femtoscopic correlation lengths, also referred to as HBT radii [11, 12], using the Correlation AfterBurner (CRAB) code $[13,14]$. A chi-squared statistic is used to determine the best fit initial state parameters and associated uncertainties for the measured results and errors.

\section{Model Configuration}

To generate the model results, the initial temperature distribution was calculated by integrating over 10,000 TGlauber participant nucleon $\left(N_{\text {part }}\right)$ distribution, in which the x-axis was aligned along the participant eccentricity axis for each event. The initial temperature (labeled by the equivalent temperature for a central collisiosn) was varied in steps of $5 \mathrm{MeV}$ in the range $270-315 \mathrm{MeV}$ for comparisons to pion spectra, and in steps of $20 \mathrm{MeV}$ over the range $260-380 \mathrm{MeV}$ for femtoscopic raddi and flow comparisons. The initial state was prepared with pre-equilibrium flow calculated in accordance with [8]. To study the sensitivity to the equation of state, we ran two separate evolutions for each initial parameter set: the the QCD-inspired Equation of State based upon the work of Laine and Schroeder [15] that interpolates between the hadronic resonance model and the perturbative calculation, and the LQCD equation of state calculated with a stout fermion action by the Budapest-Wuppertal group [16]. We have also begun testing the model with the HotQCD equation of state [17], but these results are not yet complete. A comparison of several current equations of state is shown in Fig 1. Note that the current parameterization for the hisq action 

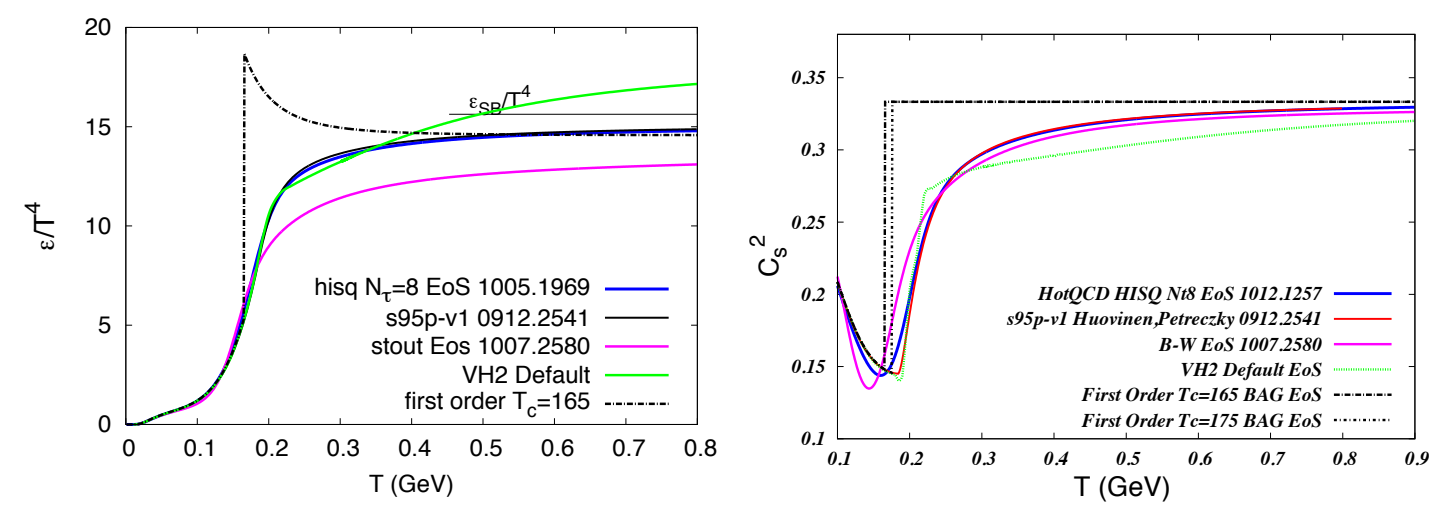

Figure 1: Energy density (left) and speed of sound squared (right) for several different equation of state calculations. Hisq, stout, and s95p-v1 are fits to LQCD calcualtions, the VH2 default is based on [15] and the first order EoS is calculated from the hadron resonance gas below and bag model above using a critical temperature of $165 \mathrm{MeV}$.

is quite similar to the s95p-v1 fit by Huovinen and Petreczky [18]. The default EoS for VH2 eventually rises above the Stefan-Boltzman value for the energy density, but is otherwise is quite consistent with the LQCD calculations for the initial temperatures employed here. Conversely, the stout action energy density falls below all other calculations for temperatures above the transition. For this reason the initial temperature range was shifted upwards by $20 \mathrm{MeV}$ for all calculations using the stout action.

The hydrodynamic evolution in $\mathrm{VH} 2$ was stopped when the temperature of a given cell fell below a specified freeze-out value, nominally set to $165 \mathrm{MeV}$. The final energy densities were converted to final state particles following the prescription of Cooper and Frye [19] with corrections for the shear viscosity implemented according to the method developed by Pratt and Torrieri [20]. For each set of CHIMERA parameters, a set of 5,000 events were generated in the OSCAR-97 format [21] to compare to the measured particle spectra. For comparisons to elliptic flow and radii, the total number of events was increased to 20,000 in order to achieve smaller statistical errors in the model results prior to fitting.

UrQMD v2.3 was used to calculate the evolution of the hadronic transport stage. The final state particle distributions from UrQMD were then used to construct the observables that can be directly compared to published experimental data. In this work we restricted our comparison to transverse momentum spectra, the second coefficient of the transverse momentum anisotropy with respect to the event reaction plane $v_{2}$, and the Bertsch-Pratt femtoscopic radii, $R_{\text {long }}, R_{\text {side }}, R_{\text {out }}$. The transverse momentum spectra are calculated as invariant cross-sections using bins of $0.1 \mathrm{GeV} / \mathrm{c}$ in the range $0.2-1.5 \mathrm{GeV} / \mathrm{c}$ within the rapidity interval of $|y|<0.5$. The elliptic flow was calculated within the same transverse momentum region. We used CRAB to generate the three-dimensional femtoscopic correlation functions, including the strong interaction and statistical interference. The correlation functions were binned in $0.2 \mathrm{GeV}$ intervals, and the correlation fits were performed directly on the correlation weights, to avoid the time and cpu-consuming process of constructing an event mixed background for each momentum bin. The binned results for $v_{2}$ and the hbt radii 

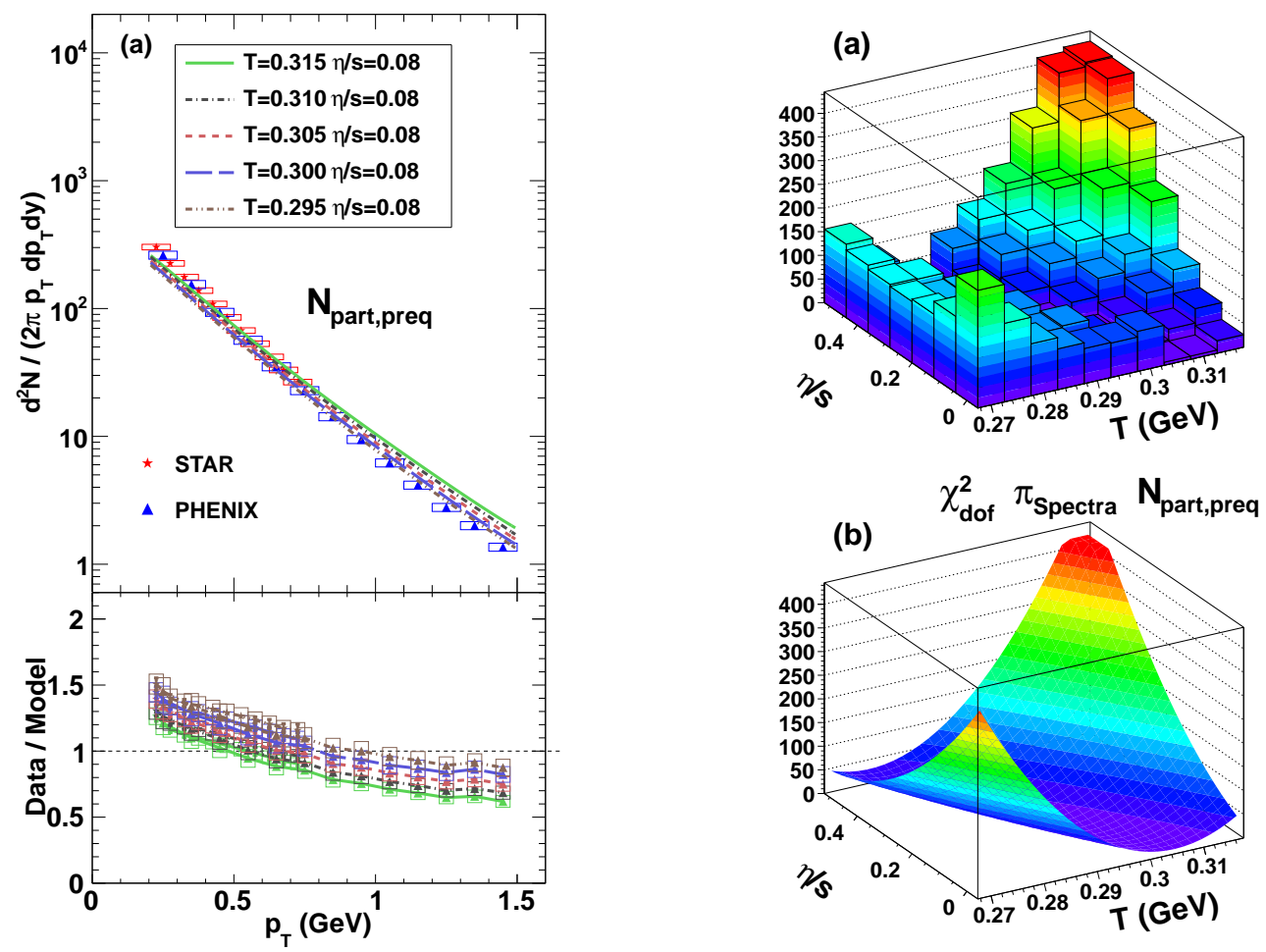

Figure 2: Model results for pion spectra compared to measurements by PHENIX and STAR (left), $\chi_{n d f}^{2}$ evaluations (top-right), and paraboloid fits (bottom-right).

were then fit to third order Chebyshev polynomials to simplify the $\chi^{2}$ evaluation, and a fifth order polynomial multiplied by an exponential distribution was used to fit the spectra [6].

\section{Results}

Comparisons to the spectra measured by PHENIX [22] and STAR [23] are shown in the left panel of Fig. 2. The spectra from CHIMERA are harder than the measurements, however, the $\chi_{n d f}^{2}$ values shown the the valley of the top right panel are in the range of 10-20, due to the systematic errors. To incorporate the systematic errors when evaluating the chi-squared statistic, we follow the procedure defined in [24]. Because it is generally not feasible to calculate a full co-variance matrix for the systematic errors, the authors of [24] make a set of simplifying assumptions that correspond to different types of systematic errors. For this analysis we assume all systematic errors assumed to be of type B, in which the systematic errors are assumed to be fully correlated within a single experimental analysis. The bottom-right panel if Fig. 2 shows a paraboloid fit to the $\chi_{n d f}^{2}$ distribution that will eventually be used to establish confidence contours.

For the $v_{2}$ we compare to two sets of measurements by PHENIX in this centrality range, a combined analysis of pions and kaons [25], and a recent analysis of pion $v_{2}$ at higher $p_{T}$ [26], as well as a measurement by STAR [27]. The top-left panel shows the pion elliptic flow compared to STAR [27] and higher transverse momentum data from PHENIX [26] and the bottom-left panel 

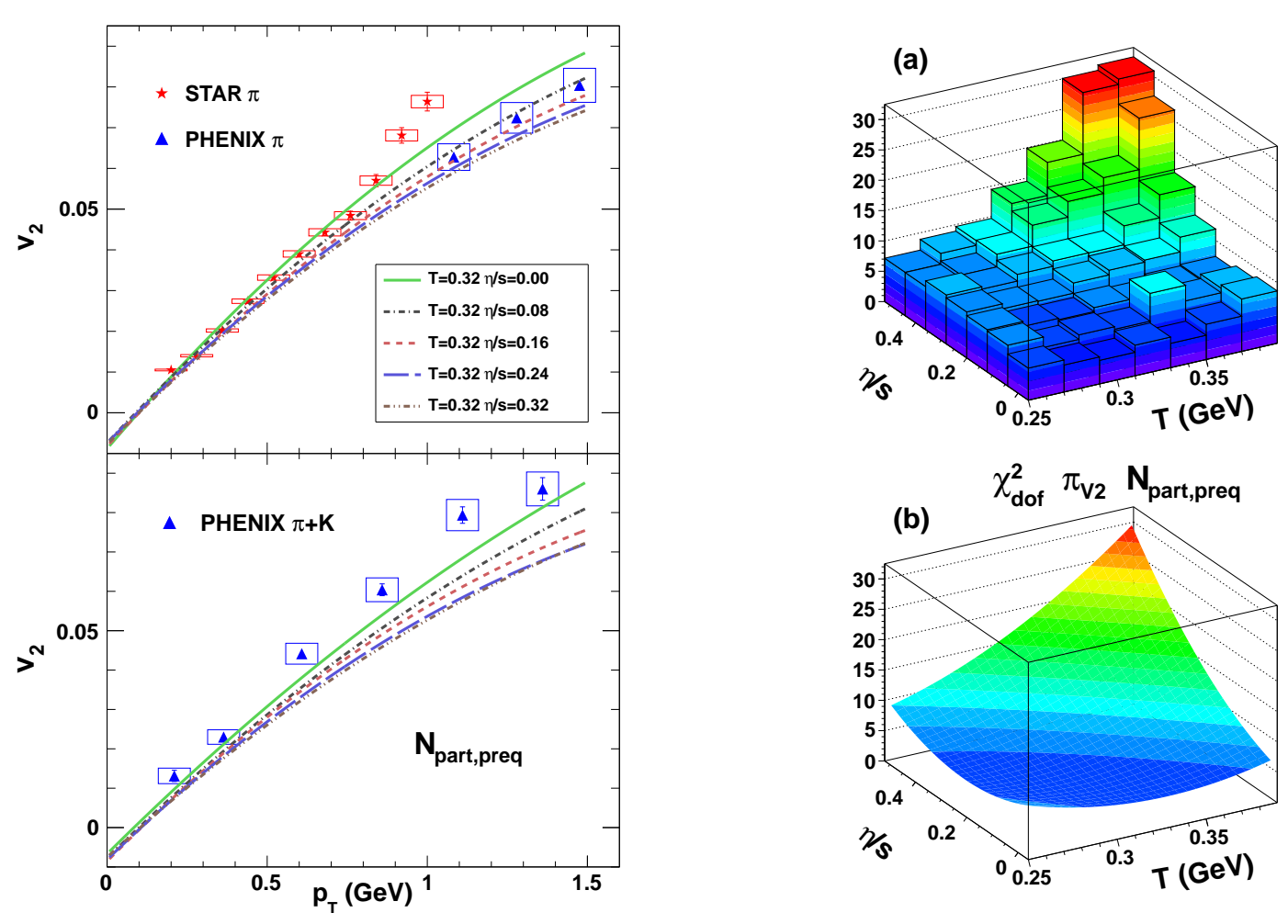

Figure 3: Model results for pion and kaon $v_{2}$ compared to measurements by PHENIX and STAR (left), $\chi_{n d f}^{2}$ evaluations (top-right), and paraboloid fits (bottom-right).

shows a comparison the combined elliptic flow for pions and kaons measured by PHENIX [25]. As with the spectra, the right panels show the $\chi_{n d f}^{2}$ distribution and paraboloid fit. The comparison for femtoscopic radii, $\chi_{n d f}^{2}$ distrbitution, and paraboloid fit are shown in Fig. 4. These results confirm that the so-called "hbt-puzzle" solution of pre-equilibrium flow, cross-over equation of state, and hadronic cascade continues to work in $2 \mathrm{D}+1$ dimensional hydrodynamics [28]. Reasonable $\chi_{n d f}^{2}$ values are achieved for a wide range of viscosities and initial temperatures just under $300 \mathrm{MeV}$.

\section{Discission}

The paraboloid fits are summarized in Fig. 5 in the form of confidence contours. The dashed and solid lines denote where paraboloid fit increases by one and two, respectively, and the minimum values for HBT radii, Spectra, and $v_{2}$ are listed in the upper right quadrant in each panel. The negative value for the $v_{2}$ fitted value for qcdIEOS is likely due to the larger fluctuation in the $\chi_{n d f}^{2}$ values for $v_{2}$ and is not physically meaningful except as an overall indication that the $\chi_{n d f}^{2}$ values for all measurements are below ten. The shaded region denotes the region in which all three measurements overlap within the second contour. These results are consistent with earlier conclusions that $\eta / s<2.4[1,29]$. The primary sensitivity to the equation of state is in the value of the initial temperature, shown here to be $20 \mathrm{MeV}$ higher for the Budapest-Wuppertal equation of state calculated with the stout fermion action. 

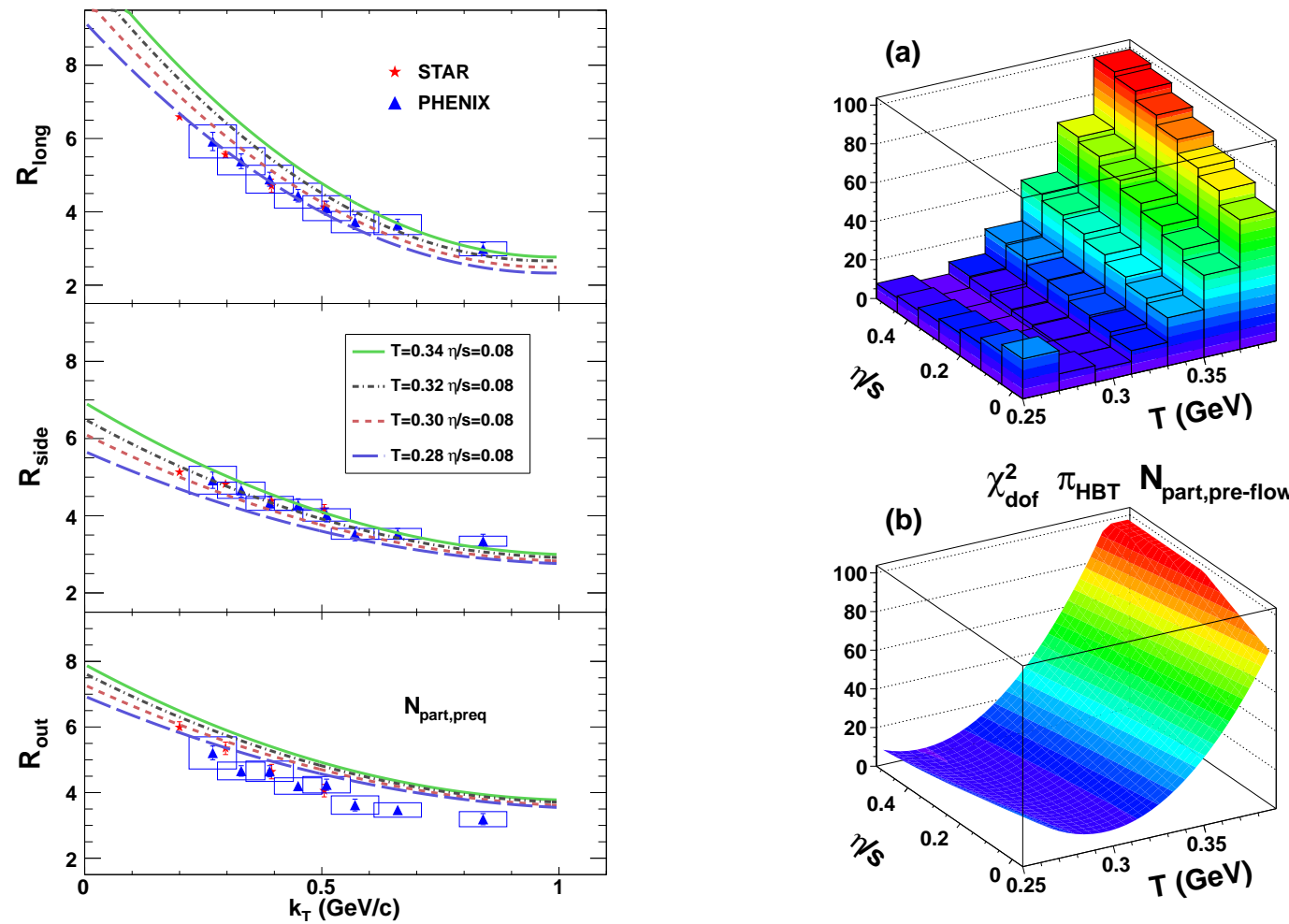

Figure 4: Model results for pion and radii compared to measurements by PHENIX and STAR (left), $\chi_{n d f}^{2}$ evaluations (top-right), and paraboloid fits (bottom-right).
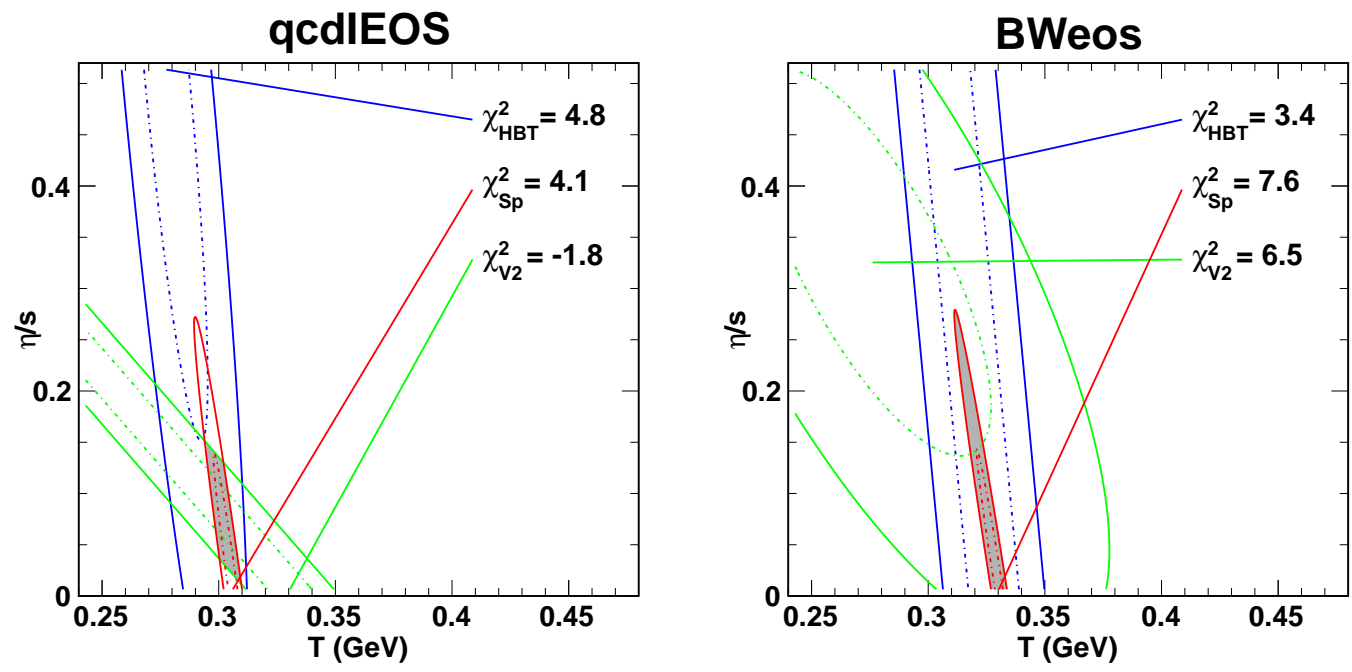

Figure 5: Confidence contours for paraboloid fits to the $\chi_{n d f}^{2}$ distributions for the default $\mathrm{VH} 2$ equation of state, qcdIEOS (left) and the LQCD equation of state calculated by the Budapest-Wuppertal collaboration (right). The dashed and solid lines denote where paraboloid fit increases by one and two, respectively, and the minimum values for HBT radii, Spectra, and $v_{2}$ are denoted in the upper right quadrant in each panel. 
In the future, we plan to extend this study by using the HotQCD equation of state calculated with the hisq action, as well as a first order equation of state in order to explore sensitivity to a change in the equation of state at higher baryon density that may occur for lower energy collisions. We also plan to replace the $\mathrm{VH} 2$ hydrodynamic code with a relativistic viscous hydrodynamic code that can incorporate initial state fluctuations.

\section{References}

[1] M. Luzum and P. Romatschke, Phys. Rev. C 78 (2008) 034915.

[2] H. Song, S. Bass, and U. Heinz, Phys. Rev. C 83 (2011) 024912.

[3] T. Kodama, C. E. Aguiar, T. Osada, and Y. Hama, J. Phys. G 27 (2001) 557.

[4] B. Schenke, S. Jeon, and C. Gale, Phys. Rev. C 82 (2010) 014903.

[5] Z. Qiu and U. Heinz, Phys. Rev. C 84 (2011) 024911.

[6] R. A. Soltz, I. Garishvili, M. Cheng, B. Abelev, A. Glenn, and others Phys.Rev. C87 (2013) 044901.

[7] PHOBOS Collaboration, B. Alver et al., Phys. Rev. C 77 (2008) 014906.

[8] J. Vredevoogd and S. Pratt, Phys. Rev. C 79 (2009) 044915.

[9] S. A. Bass et al., Prog. Part. Nucl. Phys. 41 (1998) 225.

[10] M. Bleicher et al., J. Phys. G 25 (1999) 1859.

[11] R. Hanbury Brown and R. Q. Twiss, Philos. Mag. 45 (1954) 663.

[12] G. Goldhaber, S. Goldhaber, W. Lee, and A. Pais, Phys. Rev. 120 (1960) 300.

[13] S. Pratt, T. Csörgó, and J. Zimányi, Phys. Rev. C 42 (1990) 2646.

[14] D. Brown et al., Phys. Rev. C 72 (2005) 054902.

[15] M. Laine and Y. Schröder, Phys. Rev. D 73 (2006) 085009.

[16] S. Borsányi et al., J. High Energ. Phys. 2010 (2010) 77.

[17] HotQCD Collaboration, A. Bazavov et al., Phys. Rev. D 80 (2009) 014504.

[18] P. Huovinen and P. Petreczky, 837 (2010) 26.

[19] F. Cooper and G. Frye, Phys. Rev. D 10 (1974) 186.

[20] S. Pratt and G. Torrieri, Phys. Rev. C 82 (2010) 044901.

[21] https://karman.physics.purdue.edu/OSCAR/index.php, 1997.

[22] PHENIX Collaboration, S. S. Adler et al., Phys. Rev. C 69 (2004) 034909.

[23] STAR Collaboration Collaboration, J. Adams et al., Phys. Rev. Lett. 92 (2004) 112301.

[24] PHENIX Collaboration Collaboration, A. Adare et al., Phys. Rev. C 77 (2008) 064907.

[25] PHENIX Collaboration Collaboration, S. Adler et al., Phys. Rev. Lett. 91 (2003) 182301.

[26] PHENIX Collaboration Collaboration, A. Adare and other, Phys. Rev. C 85 (2012) 064914.

[27] STAR Collaboration Collaboration, J. Adams et al., Phys. Rev. C 72 (2005) 014904.

[28] S. Pratt, Phys. Rev. Lett. 102 (2009) 232301.

[29] H. Song, S. A. Bass, U. Heinz, T. Hirano, and C. Shen, Phys. Rev. Lett. 106 (2011) 192301. 